\title{
Ultra-faint Lyman Alpha Emitters with MUSE
}

\author{
Michael V. Maseda and the MUSE GTO Consortium \\ Leiden Observatory, Leiden University, Postbus 9513, NL-2300RA, Leiden, the Netherlands \\ email: maseda@strw.leidenuniv.nl
}

\begin{abstract}
Using an ultra-deep, untargeted survey with the MUSE integral field spectrograph on the ESO Very Large Telescope, we obtain spectroscopic redshifts to a depth never explored before: galaxies with observed magnitudes $m>30-32$. Specifically, we detect objects via Lyman$\alpha$ emission at $2.9<z<6.7$ without individual continuum counterparts in areas covered by the deepest optical/near-infrared imaging taken by the Hubble Space Telescope, the Hubble Ultra Deep Field. In total, we find more than 100 such objects in 9 square arcminutes at these redshifts, also including a number of sources that are visible only in the HST band that contains Lyman$\alpha$. Detailed HST and IRAC stacking analyses confirm the Lyman- $\alpha$ emission as well as the $1216 \AA$ breaks, faint UV continua $\left(M_{\mathrm{UV}} \sim-15\right)$, and optical emission lines: these objects are the faintest spectroscopically-confirmed galaxies at high- $z$. The blue UV continuum slopes and measurements/limits on the equivalent widths of Lyman- $\alpha$, which in some cases exceeds $300 \AA$, are consistent with ages $<10 \mathrm{Myr}$, metallicities $<5 \%$ solar, and stellar masses $<10^{7-8}$ solar masses. The nature of these types of objects is intriguing as they could be the faint star-forming sources of Reionization and could represent the initial (strong) phase of stellar mass growth in galaxies.
\end{abstract}

Keywords. galaxies: dwarf, galaxies: high-redshift

\section{Introduction}

Until recently, large samples of star forming galaxies at $z>1$ were created exclusively via color selections. These selections, such the Lyman break (Steidel et al. 2003), BzK (Daddi et al. 2004), and BX/BM selections (Steidel et al. 2004), suffer variously from issues of incompleteness and contamination from lower- $z$ interlopers, as well as preferentially selecting galaxies with certain dust properties (Ly et al. 2011). When the selection criteria become stricter to avoid some of these issues, then the selection itself becomes more biased. For example, the Lyman break selection requires detections in several bands redward of the actual break in the spectral energy distribution (SED), potentially biasing against low-mass bursts with strong emission lines but little stellar continuum light.

To circumvent this issue, unbiased, un-targeted spectroscopic surveys are required. Previous efforts to do this utilized long-slit spectra taken over blank regions of sky (e.g. Rauch et al. 2008), in some cases centered on the caustics of strong gravitationally lensing systems (e.g. Ellis et al. (2001); Stark et al. (2007)). However, the area covered by these types of surveys is necessarily small ( $\lesssim 0.2$ square arcminutes), and the ability to measure line fluxes and slit loss corrections when no deep continuum imaging exists is problematic. Slitless surveys taken with prisms (e.g. Salzer et al. (2000)) or grisms (e.g. Momcheva et al. 2016) can overcome these issues, but at the cost of a higher background flux level.

The Multi-Unit Spectroscopic Explorer (Bacon et al. 2010), however, overcomes many of these issues. Its image slicer design provides low-background optical spectroscopy simultaneously over a large field-of-view (1 square arcminute). This makes MUSE 
well-suited to undertake mosaicked spectroscopic surveys as a compliment to wide-field imaging surveys: in the first deep MUSE data cubes covering the Hubble Deep Field South (HDFS), 189 individual objects have confirmed redshifts in 27 hours of spectroscopy (Bacon et al. 2015).

Due to the un-targeted nature of MUSE spectroscopy, 26 objects in the HDFS have detectable Lyman- $\alpha$ emission lines at $2.9<z<6.7$ with no counterpart in the Hubble Space Telescope (HST) WFPC2 imaging. The WFPC2 observations of the HDFS reach a 5- $\sigma$ limiting AB magnitude of 29.1 in F606W and 28.5 in F814W (Casertano et al. 2000). The Hubble Ultra Deep Field (UDF), however, reaches depths more than one magnitude deeper in nine HST filters spanning the optical to near-IR (Illingworth et al. 2013) and is the target of deeper, 30-hour observations with MUSE (Bacon et al. 2017). By combining the MUSE spectroscopy with the HST imaging in the UDF, we can search for the faintest galaxies in terms of both emission line fluxes as well as continuum magnitudes.

\section{Observations and Results}

In order to identify emission lines without relying on a photometric detection, we use the ORIGIN software (Bacon et al. 2017, D. Mary et al. in prep) to find emission lines in the reduced MUSE UDF data cubes. The individual MUSE data cubes are combined into a 9 square arcminute "mosaic" with a depth of 10 hours and a single, 1 square arcminute pointing with a depth of 30 hours. All detected sources in the full dataset have redshifts determined by using a combination of automated tools and expert identifications (see Inami et al. 2017 for details). Lyman- $\alpha$ emitters (LAEs) can often be identified due to the characteristic asymmetry, resolvable at the spectral resolution of MUSE. Additionally, lower-redshift solutions can ruled out based on multiple optical emission line non-detections due MUSE's broad wavelength coverage.

We place $0.4^{\prime \prime}$ apertures in all HST bands centered on the MUSE emission line centroid. The local background level in each band is calculated by measuring the standard deviation of the fluxes in 250 identical apertures spread randomly within a $10^{\prime \prime} \times 10^{\prime \prime}$ cutout centered on the object, while masking other sources according to the imaging segmentation maps. An object is considered "undetected" if there are no $>5-\sigma$ detections in any of the HST bands. In total we identify 102 MUSE LAEs that have no counterpart in the HST imaging.

As these galaxies are individually undetected, we must rely on photometric stacking in order to learn about their continuum properties. We split the galaxies into three redshift bins corresponding to a single HST filter probing the restframe UV $(\sim 1600 \AA)$. Figure 1 shows clear detections in the band(s) that contain Lyman- $\alpha$ and the (blue) UV continuum. Combined with non-detections in bands bluewards of Lyman- $\alpha$, these objects fulfill the photometric criteria for the aforementioned Lyman break galaxies. This provides independent, photometric evidence of the Lyman- $\alpha$ identification. Based on the aperture magnitude of the UV continuum, these galaxies have $M_{\mathrm{UV}}$ values of $\sim-15$, more than 3 magnitudes fainter than continuum-selected samples in the field: these are the faintest spectroscopically-confirmed galaxies at high- $z$ discovered to date (Maseda et al. 2018).

The detectable Lyman- $\alpha$ emission and extremely faint UV continuum implies that the Lyman- $\alpha$ equivalent widths (EWs) are extremely high, typically $>300 \AA$ (M. Maseda et al. in prep.). These extreme EWs can only be produced by young, low-metallicity stellar populations. Stellar population models can predict the evolution in the EW of Lyman- $\alpha$ as a function of age, metallicity, and the star formation history (SFH) of the galaxy. These models also predict the evolution in the observed UV continuum slopes $(\beta)$. By combining measurements of the Lyman- $\alpha$ EW and $\beta$ from the stacks, we can constrain the age-metallicity-SFH parameter space.

In Figure 2 we show how each stack's constraints on $\beta$ and Lyman- $\alpha$ EW translates into an age, metallicity, and stellar mass (age convolved with $\mathrm{SFH}$ ) using the models of 

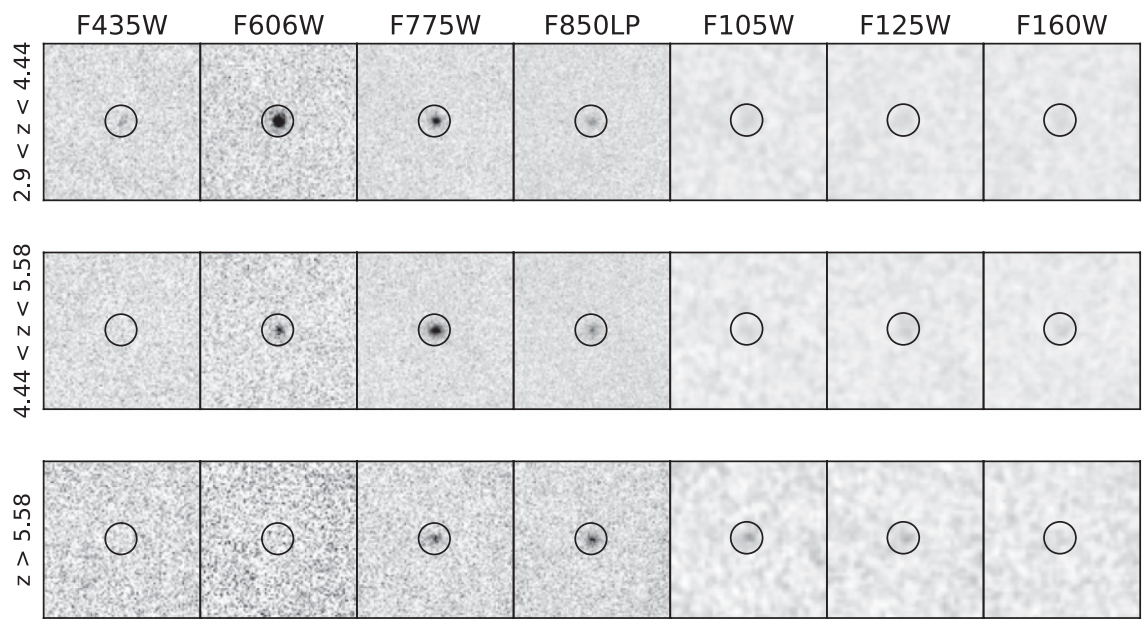

Figure 1. HST imaging stacks for the undetected LAEs in three different redshift bins. Lyman$\alpha$ emission is visible in the $F 606 W$ (top), $F 606 W$ and $F 775 W$ (middle), and $F 775 W$ and $F 850 L P$ bands (bottom), in line with the redshift determined by MUSE. All of these photometric stacks fulfill the standard "dropout" selection criteria for LBGs at these redshifts.

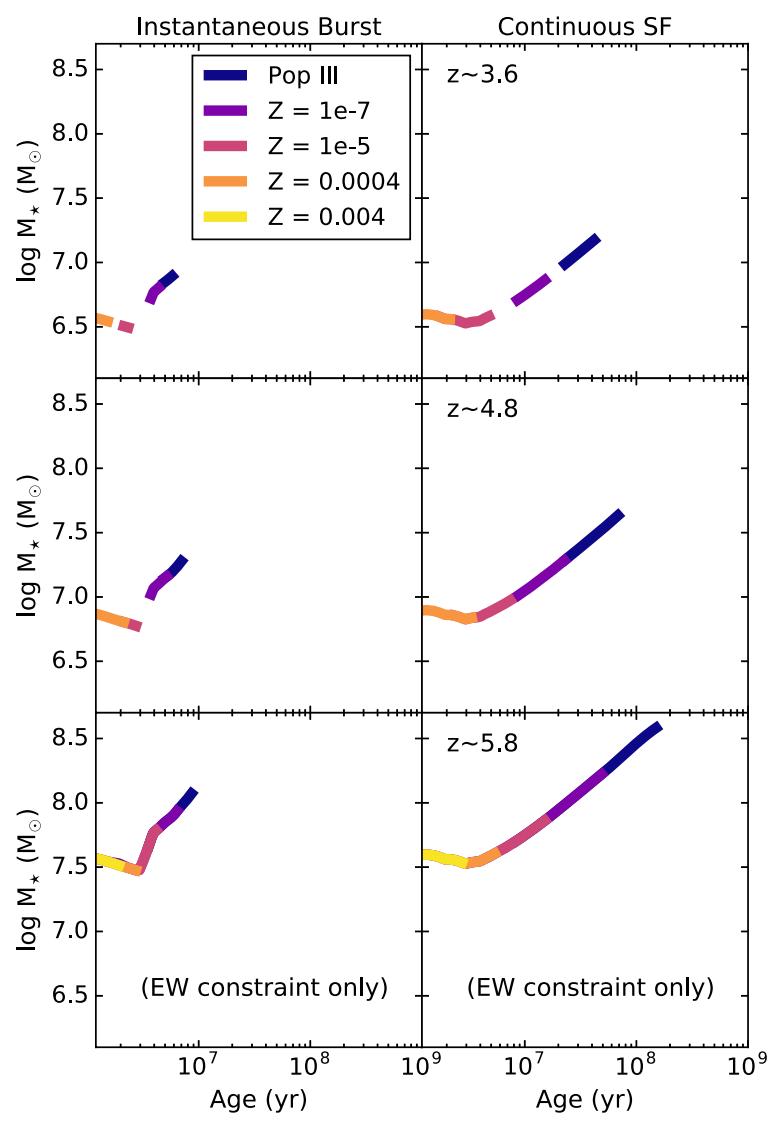

Figure 2. Stellar mass estimates as a function of age, metallicity, and star formation history (burst or continuous) based on the observed Lyman- $\alpha$ EWs and UV continuum slopes $(\beta)$, using the models of Raiter et al. 2010. The highest stellar masses can be achieved with the lowest metallicities and continuous star formation, and in general the metallicity must be < $0.0004\left(\sim 5 \% \mathrm{Z}_{\odot}\right)$ when both the $\mathrm{EW}$ and $\beta$ are constrained. 
Raiter et al. 2010. Although $\beta$ is not constrained for the $z>5.58$ stack due to the lack of continuum bands probing the rest-UV at these redshifts, in general we need metallicities $<0.0004\left(\sim 5 \% \mathrm{Z}_{\odot}\right)$, burst ages $<10 \mathrm{Myr}$, and stellar masses $<10^{8} \mathrm{M}_{\odot}$. This implies these are galaxies undergoing their first significant episode of star formation.

\section{Future Outlook}

Further spectroscopic work is required to fully characterize the stellar populations in these ultra-faint LAEs. In particular, detections of restframe-optical emission lines will provide comparable information about the metallicity and age of the galaxy. At these redshifts spectroscopic observations of the restframe-optical are challenging as these features are shifted further into the near-IR and are difficult or impossible to observe from the ground. However, at certain redshifts these features are located in broadband photometry from e.g. Spitzer/IRAC. Since the redshifts are known a priori from MUSE, any photometric excess in the IRAC photometry can be attributed to e.g. H $\alpha$ emission. With a measurement of $\mathrm{H} \alpha$ and Lyman- $\alpha$, we can measure the ionizing photon production efficiency $\left(\xi_{\text {ion }}\right)$, which is crucial to understand in the context of cosmic Reionization (e.g. Robertson et al. 2015).

At $3.829<z<4.955, \mathrm{H} \alpha$ is in IRAC's Channel 1 and Lyman- $\alpha$ is probed by MUSE. For a sample of 40 HST-faint LAEs, we create a stack of the IRAC Ch. 1 photometry and find a $>5-\sigma$ detection within a $1.8^{\prime \prime}$ aperture. If the $\mathrm{H} \alpha$ emission is confirmed to be real, e.g. with JWST/NIRSpec, then the implied high equivalent width makes these galaxies even more extreme than the "Extreme Emission Line Galaxies" discovered at $z \sim 1-2$ in slitless HST grism spectroscopy (e.g. Maseda et al. 2013, 2014), which have values of $\xi_{\text {ion }}$ up to $\sim 25.8$ (Tang et al. 2018 in press). An abundant population of these galaxies, as revealed with our MUSE survey, is likely to have made a significant contribution to cosmic Reionization. Further work is required to quantify this contribution.

\section{References}

Bacon, R., Accardo, M., Adjali, L., et al. 2010, SPIE, 773508

Bacon, R., Brinchmann, J., Richard, J., et al. 2015, A\&A, 575, A75

Bacon, R., Conseil, S., Mary, D., et al. 2017, A\& $A$, 608, A1

Casertano, S., de Mello, D., Dickinson, M., et al. 2000, AJ, 120, 2747

Daddi, E., Cimatti, A., Renzini, A., et al. 2004, ApJ, 617, 746

Ellis, R., Santos, M. R., Kneib, J.-P., et al. 2001, ApJ, 560, L119

Illingworth, G. D., Magee, D., Oesch, P. A., et al. 2013, ApJS, 209, 6

Inami, H., Bacon, R., Brinchmann, J., et al. 2017, A\&A, 608, A2

Ly, C., Malkan, M. A., Hayashi, M., et al. 2011, ApJ, 735, 91

Maseda, M. V., van der Wel, A., da Cunha, E., et al. 2013, ApJ, 778, L22

Maseda, M. V., van der Wel, A., Rix, H.-W., et al. 2014, ApJ, 791, 17

Maseda, M. V., Bacon, R., Franx, M., et al. 2018, ApJ, 865, L1

Momcheva, I. G., Brammer, G. B., van Dokkum, P. G., et al. 2016, ApJS, 225, 27

Raiter, A., Schaerer, D., \& Fosbury, R. A. E. 2010, A\&GA, 523, A64

Rauch, M., Haehnelt, M., Bunker, A., et al. 2008, ApJ, 681, 856

Robertson, B. E., Ellis, R. S., Furlanetto, S. R., et al. 2015, ApJ, 802, L19

Salzer, J. J., Gronwall, C., Lipovetsky, V. A., et al. 2000, AJ, 120, 80

Stark, D. P., Ellis, R. S., Richard, J., et al. 2007, ApJ, 663, 10

Steidel, C. C., Adelberger, K. L., Shapley, A. E., et al. 2003, ApJ, 592, 728

Steidel, C. C., Shapley, A. E., Pettini, M., et al. 2004, ApJ, 604, 534

Tang, M., Stark, D., Chevallard, J., et al. 2018, arXiv e-prints, arXiv:1809.09637 


\section{Discussion}

S. WUYTs Based on the observed number counts of these galaxies, can you derive an estimate for the total number density of galaxies at this UV magnitude? This would be an independent measurement of (part of) the UV luminosity function at these redshifts.

MASEDA We have not done this yet, but in theory we do have a well-defined selection function based on Lyman- $\alpha$ flux. Based on this we could estimate the volume density of these sources that have $M_{\mathrm{UV}} \sim-15$. However this is likely to be a lower limit since there should be a significant population of sources at a similar continuum magnitude that do not have such high Lyman- $\alpha$ EWs and hence would be unobservable in our survey. Additionally, this sample was selected for purity rather than completeness, as we manually remove any sources that are very close to HST detections even if it is clearly at a different redshift.

E. DA Cunha At some level do we not expect all galaxies at such faint magnitudes to be undergoing some kind of bursty episode of star formation?

MASEDA Bursty star formation does appear to be the norm at low masses and low metallicities in the early Universe. However, the UV magnitude is not a direct probe of the stellar mass of a galaxy, and hence for a fixed faint UV magnitude we could also expect a number of more massive, older galaxies in addition to the young starbursts. Presumably these older galaxies were once bursty in the past, but it is difficult to say how bright they were. Likewise, it is difficult to know what these high-EW LAEs will look like in a few Myrs. 